\title{
An Improved Theoretical Approach to Study Electromagnetic Waves through Fiber Bragg Gratings
}

\author{
Pedro Pereyra \\ Departamento de Ciencias Básicas, UAM Azcapotzalco, 02200 Mexico City, Mexico \\ Correspondence should be addressed to Pedro Pereyra; pereyrapedro@gmail.com
}

Received 1 September 2016; Revised 5 December 2016; Accepted 6 December 2016; Published 10 January 2017

Academic Editor: Joseph S. Poon

Copyright (C) 2017 Pedro Pereyra. This is an open access article distributed under the Creative Commons Attribution License, which permits unrestricted use, distribution, and reproduction in any medium, provided the original work is properly cited.

\begin{abstract}
We show that using the theory of finite periodic systems we obtain an improved approach to calculate transmission coefficients and transmission times of electromagnetic waves propagating through fiber Bragg gratings. We discuss similarities, advantages, and differences between this approach and the well known less accurate one coupled mode approximation and the pseudo-Floquet Mathieu functions approach.
\end{abstract}

\section{Introduction}

A complete theoretical description of the electromagnetic waves motion through fiber Bragg gratings (FBG) remains to be achieved. Despite the numerous numerical calculations and simulations (see, e.g., $[1,2]$ ) the full analytical solution of the wave equation in the presence of a periodic modulation of the refractive index is still an open problem. In the last years, a large number of fiber Bragg gratings devices were developed for different types of applications, among others, for optical communications devices, tunable wavelength filters and temperature, and pressure sensors [3-8]. In the theoretical approaches proposed to study these systems, one has also to deal, besides of the spatial modulation of the refractive index, with model limitations and the finiteness of the actual Bragg systems. In the small modulation amplitude limit, the wave equation becomes a Mathieu equation, and one has to face divergency problems in the numerical evaluation of these functions. For standard wavelengths of FBG, written with ultraviolet lasers, $1 \mathrm{~cm}$ fiber contains thousands of refractive-index modulations. An extensive literature has been published on coupled modes approximation [9-13], and a simplified one-dimensional model of two counterrunning and synchronous modes was introduced by Kogelnik and Shank $[14,15]$. This single mode approximation, called also the one coupled mode approximation (OCMA), has been a useful and simplified approach [16]. However this model works well only for frequencies in the neighborhood of the Bragg frequency $\omega_{B}$. For this reason an alternative method that will make it possible to study the transmission properties of electromagnetic waves in the whole range of frequencies is very much called for.

In this paper, we will present a theoretical approach, based on the theory of finite periodic systems [17-23] (TFPS) that will allow a precise calculation of transmission coefficients and transmission phase times through finite fiber Bragg gratings. We will show that, at least, the predictions of the well-established models are fully reproduced.

In the next sections we will outline the one coupled mode approximation (OCMA), the Mathieu functions approximation, and the theory of finite periodic systems (TFPS). We will derive the relevant results using the transfer matrix method and we will apply these results to study the evolution of longitudinal electromagnetic waves across a fiber Bragg grating. We will evaluate transmission coefficients and transmission times of specific Bragg grating fibers and compare the specific results of the well-established one coupled mode approximation with those of the theory of finite periodic systems (TFPS) and the Mathieu functions approximation. To overcome divergencies using Mathieu function we will also show results obtained in a combined approach of Mathieu functions and the TFPS. 


\section{Definition of the Fiber Bragg Grating}

Suppose we have a Bragg grating fiber of length $L_{o}$ and refractive index (we use this notation to avoid confusion with the number of unit cells, $n$, in the fiber Bragg grating):

$$
r(z)=r_{o}\left(1+2 V_{o} \cos \frac{2 \pi z}{\Lambda}\right)
$$

where $\Lambda$ is the grating period $\left(\Lambda=\lambda / 2 r_{o}\right), r_{o}$ is the effective refractive index, $\lambda$ is the vacuum wave length, and $V_{o}$ is the modulation amplitude. This is a typical periodic system. The space-time evolution of electromagnetic fields in these systems is governed by the wave equation.

$$
\frac{\partial^{2} E}{\partial z^{2}}=\frac{r^{2}(z)}{c^{2}} \frac{\partial^{2} E}{\partial t^{2}}
$$

To study the space-time evolution of electromagnetic waves, we shall, first, recall the one coupled mode approximation and obtain the transmission coefficient and the transmission time as functions of the frequency. We will then obtain the same quantities using the Mathieu functions and, at the end, using the theory of finite periodic systems.

2.1. The FBG in the One Coupled Mode Approximation. In the one coupled mode approximation and following Erdogan's notation, the system of equations for the left and right moving field amplitudes, dominant at the Brag reflection frequency, is written as

$$
\begin{aligned}
& \frac{\partial u}{\partial z}=\eta u+\kappa v, \\
& \frac{\partial v}{\partial z}=-\eta v-\kappa u .
\end{aligned}
$$

If we define the wave vector

$$
f(z)=\left(\begin{array}{l}
u(z) \\
v(z)
\end{array}\right)
$$

the system of (3) can be written as

$$
\frac{d}{d z} f=\left(\begin{array}{cc}
\eta & \kappa \\
-\kappa & -\eta
\end{array}\right) f
$$

whose solution, for $\eta$ and $\kappa$ independent of $z$, is

$$
f(z)=e^{\left(\begin{array}{cc}
\eta & \kappa \\
-\kappa & -\eta
\end{array}\right) z}
$$

It is easy to verify that the transfer matrix

$$
M\left(z_{2}, z_{1}\right)=e^{\left(\eta \sigma_{z}-i \kappa \sigma_{y}\right)\left(z_{2}-z_{1}\right)}
$$

with $\sigma_{z}$ and $\sigma_{y}$ being the Pauli matrices, satisfies the relation

$$
\left(\begin{array}{l}
u\left(z_{2}\right) \\
v\left(z_{2}\right)
\end{array}\right)=M\left(z_{2}, z_{1}\right)\left(\begin{array}{l}
u\left(z_{1}\right) \\
v\left(z_{1}\right)
\end{array}\right) .
$$

Expanding the exponential function, the transfer matrix in (7) becomes [16]

$$
M\left(z_{2}, z_{1}\right)=\left(\begin{array}{cc}
\alpha_{G} & \beta_{G} \\
-\beta_{G} & \delta_{G}
\end{array}\right),
$$

where

$$
\begin{aligned}
& \alpha_{G}=\cosh K\left(z_{2}-z_{1}\right)+\frac{\eta}{K} \sinh K\left(z_{2}-z_{1}\right), \\
& \beta_{G}=\frac{\kappa}{K} \sinh K\left(z_{2}-z_{1}\right), \\
& \delta_{G}=\cosh K\left(z_{2}-z_{1}\right)-\frac{\eta}{K} \sinh K\left(z_{2}-z_{1}\right) .
\end{aligned}
$$

Here $K=\sqrt{\eta^{2}-\kappa^{2}}$. If we consider the parameters

$$
\begin{aligned}
& \eta=i \frac{r_{o}}{c}\left(\omega-\omega_{B}\right)=i \frac{r_{o}}{c} \delta, \\
& \kappa=i k_{B} V_{o}=i \frac{r_{o}}{c} \omega_{B} V_{o}=i \frac{r_{o}}{c} \omega_{o},
\end{aligned}
$$

the transfer matrix of a single Bragg grating of length $L_{o}=$ $z_{2}-z_{1}$ becomes

$$
\begin{aligned}
& M_{\mathrm{BG} a} \\
& =\left(\begin{array}{cc}
\cosh K L_{o}+i \frac{\delta}{\Omega} \sinh K L_{o} & i \frac{\omega_{o}}{\Omega} \sinh K L_{o} \\
-i \frac{\omega_{o}}{\Omega} \sinh K L_{o} & \cosh K L_{o}-\frac{i \delta}{\Omega} \sinh K L_{o}
\end{array}\right),
\end{aligned}
$$

with $\Omega=\sqrt{\omega_{o}^{2}-\delta^{2}}=\sqrt{\omega_{o}^{2}-\left(\omega-\omega_{B}\right)^{2}}=c K / r_{o}$. The frequency $\delta=\omega-\omega_{B}$ is known as the detuning frequency. It is worth noticing that this matrix lacks the information of the air-BG and the BG-air interfaces. To describe transmission through a finite BG, bounded by air or some other media, one needs in principle to multiply by

$$
\begin{aligned}
& M_{\mathrm{BG} a}=\frac{1}{2 r_{a}}\left(\begin{array}{cc}
r_{a}+r & r_{a}-r \\
r_{a}-r & r_{a}+r
\end{array}\right), \\
& M_{a \mathrm{BG}}=\frac{1}{2 r}\left(\begin{array}{ll}
r+r_{a} & r-r_{a} \\
r-r_{a} & r+r_{a}
\end{array}\right),
\end{aligned}
$$

on the left and right hand sides, respectively. For BG bounded by air $r_{a}=1$ and $r \simeq r_{o}$. It we take into account these matrices, the effect on the physical quantities is negligible. For this reason one can keep the manageable form (12). Given the simple relation

$$
t_{G}=\frac{1}{\alpha_{G}^{*}}
$$

between the transmission amplitude and the transfer matrix elements, the transmission coefficient and phase time of a FBG in the OCMA become

$$
\begin{aligned}
T_{G}= & \frac{1}{\cosh ^{2} K L_{o}+\left(\delta^{2} / \Omega^{2}\right) \sinh ^{2} K L_{o}}, \\
\tau_{G}= & \frac{n_{o} L_{o} T_{G}}{c} \frac{\delta^{2}}{\Omega^{2}}\left(\sinh ^{2} K L_{o}-\cosh ^{2} K L_{o}\right) \\
& +\frac{n_{o} T_{G}}{c \Omega}\left(1+\frac{\delta^{2}}{\Omega^{2}}\right) \cosh K L_{o} \sinh K L_{o} .
\end{aligned}
$$




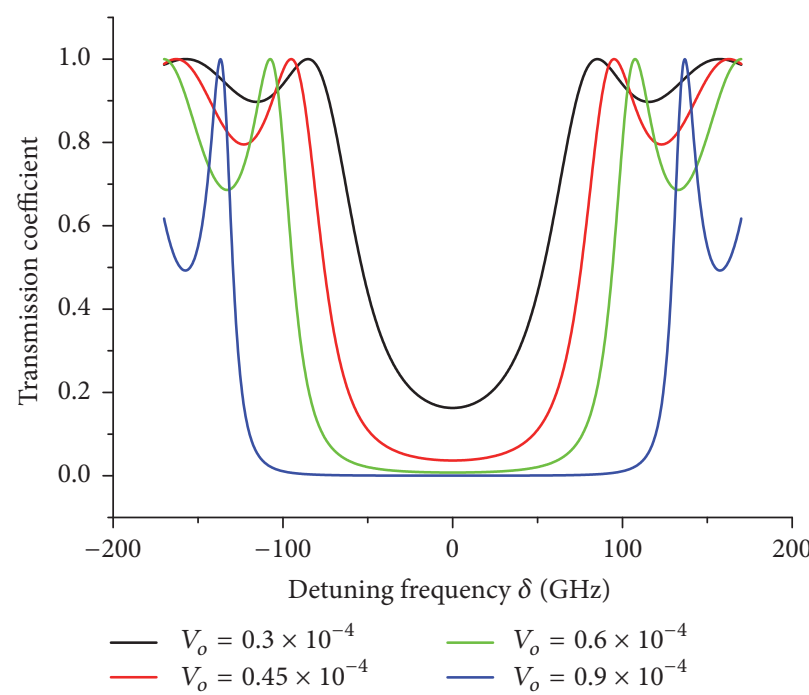

(a)

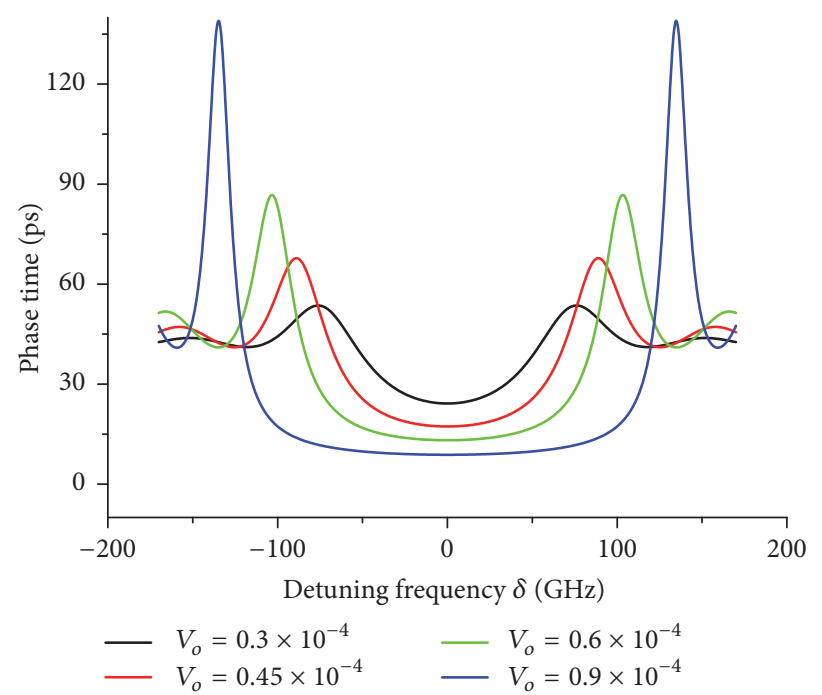

(b)

Figure 1: The transmission coefficient (a) and the phase time (b) in (15), as functions of the detuning frequency $\delta=\omega-\omega_{B}$, for different values of the modulation amplitude $V_{o}$ with $w_{B}=1.261 \times 10^{15} \mathrm{~Hz}, r_{0}=1.452$, and $L_{o}=8.5 \mathrm{~mm}$.

In the particular case of $\delta=w-w_{B}=0$ we have

$$
\begin{aligned}
& \left.T_{s} \equiv T_{G}\right|_{\delta=0}=\frac{1}{\cosh ^{2}\left(k_{B} V_{o} L_{o}\right)}, \\
& \left.\tau_{s} \equiv \tau_{G}\right|_{\delta=0}=\frac{r_{o}}{c_{o} k_{B} V_{o}} \tanh \left(k_{B} V_{o} L_{o}\right) .
\end{aligned}
$$

These expressions, written here with the notation of [24], are characteristic from the one coupled mode approach [16]. In Figures 1(a) and 1(b) we plot the transmission coefficient $T_{G}$ and the tunneling time $\tau_{G}$, as functions of the detuning frequency $\delta$, for different values of the modulation amplitude $V_{o}$. For these graphs we considered $L_{o}=8.5 \mathrm{~mm}, \omega=$ $1.261 \times 10^{15} \mathrm{~Hz}$, and $r_{o}=1.452$. Increasing the amplitude $V_{o}$, the optical gap becomes deeper and wider. At the same time, the transmission time in the gap becomes smaller. In the next sections we will present the Mathieu functions approach and the theory of finite periodic systems and, with the purpose of comparing with the results shown in Figure 1, we will calculate the same physical quantities for a system similar to that considered here.

2.2. The FBG Described by Mathieu Functions. If we come back to (2) and write the refractive index $r^{2}$, for $2 V_{o} \ll 1$, as

$$
\begin{aligned}
r^{2}(z) & =r_{o}^{2}\left(1+2 V_{o} \cos \frac{2 \pi z}{\Lambda}\right)^{2} \\
& \simeq r_{o}^{2}\left(1+4 V_{o} \cos \frac{2 \pi z}{\Lambda}\right),
\end{aligned}
$$

the wave equation becomes the Mathieu differential equation

$$
\frac{d^{2} E(z)}{d z^{2}}+\left(a_{E}-2 q_{E} \cos \frac{2 \pi z}{\Lambda}\right) E(z)=0,
$$

whose solutions $E_{1}(z)=S_{e}\left(a_{E}, q_{E} ; k_{B} z+\pi / 2\right)$ and $E_{2}(z)=$ $S_{o}\left(a_{E}, q_{E} ; k_{B} z+\pi / 2\right)$ are the well known even and odd Mathieu functions [25], with $k=\omega / c, a_{E}=k^{2} r_{o}^{2}, q_{E}=$ $2 k^{2} r_{o}^{2} V_{o}$, and $z \rightarrow z \pm \Lambda / 2$. Defining the wave vector

$$
F(z)=\left(\begin{array}{c}
E(z) \\
E^{\prime}(z)
\end{array}\right)=\left(\begin{array}{c}
A S_{e}+B S_{o} \\
A S_{e}^{\prime}+B S_{o}^{\prime}
\end{array}\right),
$$

it is possible to show that the transfer matrix

$$
W\left(z_{2}, z_{1}\right)=\left(\begin{array}{ll}
\theta & \mu \\
\nu & \chi
\end{array}\right)
$$

with

$$
\begin{aligned}
\theta\left(z_{1}, z_{2}\right) & =\frac{S_{o}^{\prime}\left(z_{1}\right) S_{e}\left(z_{2}\right)-S_{e}^{\prime}\left(z_{1}\right) S_{o}\left(z_{2}\right)}{W_{r}}, \\
\mu\left(z_{1}, z_{2}\right) & =\frac{S_{e}\left(z_{1}\right) S_{o}\left(z_{2}\right)-S_{o}\left(z_{1}\right) S_{e}\left(z_{2}\right)}{W_{r}}, \\
\nu\left(z_{1}, z_{2}\right) & =\frac{S_{o}^{\prime}\left(z_{1}\right) S_{e}^{\prime}\left(z_{2}\right)-S_{e}^{\prime}\left(z_{1}\right) S_{o}^{\prime}\left(z_{2}\right)}{W_{r}}, \\
\chi\left(z_{1}, z_{2}\right) & =\frac{S_{e}\left(z_{1}\right) S_{o}^{\prime}\left(z_{2}\right)-S_{o}\left(z_{1}\right) S_{e}^{\prime}\left(z_{2}\right)}{W_{r}}, \\
W_{r} & =S_{e}\left(z_{1}\right) S_{o}^{\prime}\left(z_{1}\right)-S_{o}\left(z_{1}\right) S_{e}^{\prime}\left(z_{1}\right),
\end{aligned}
$$

satisfies the relation

$$
F\left(z_{2}\right)=W\left(z_{2}, z_{1}\right) F\left(z_{1}\right)
$$

The transfer matrix $W\left(z_{2}, z_{1}\right)$ connects the wave vectors of Mathieu functions and their derivatives at any two points, $z_{2}$ and $z_{1}$, of an infinite periodic system. The actual Bragg 
gratings are finite with thousands of unit cells. In this case, like in the standard approach of infinite periodic systems, we assume a kind of Born-von Kármán approximation, but for the transfer matrix. More precise results will be obtained using the theory of finite periodic functions. To evaluate transmission coefficients and phase times of a BG whose length is $L_{o}$, we need to transform the matrix $W\left(L_{o}, 0\right)$ into the transfer matrix $M\left(L_{o}, 0\right)$ that connects wave vectors of propagating functions at two points just outside the BG. When the wave number of the propagating functions in vacuum is $k$, the relation between these matrices is [26]

$$
M_{G}\left(L_{o}, 0\right)=\frac{1}{2}\left(\begin{array}{cc}
1 & -i k^{-1} \\
1 & i k^{-1}
\end{array}\right) W\left(L_{o}, 0\right)\left(\begin{array}{cc}
1 & 1 \\
i k & -i k
\end{array}\right) .
$$

After multiplying, the transfer matrix that we obtain for a single Bragg grating is

$$
M_{G}\left(L_{o}, 0\right)=\left(\begin{array}{cc}
\alpha_{G} & \beta_{G} \\
\beta_{G}^{*} & \alpha_{G^{*}}
\end{array}\right)
$$

with

$$
\begin{aligned}
& \alpha_{G}=\frac{\theta\left(0, L_{o}\right)+\chi\left(0, L_{o}\right)}{2}+i \frac{k^{2} \mu\left(0, L_{o}\right)-v\left(0, L_{o}\right)}{2 k}, \\
& \beta_{G}=\frac{\theta\left(0, L_{o}\right)-\chi\left(0, L_{o}\right)}{2}-i \frac{k^{2} \mu\left(0, L_{o}\right)+\nu\left(0, L_{o}\right)}{2 k} .
\end{aligned}
$$

The transmission coefficients and the phase time are therefore given by

$$
\begin{aligned}
T_{G} & =\frac{1}{\left|\alpha_{G}\right|^{2}}=\frac{4 k^{2}}{k^{2}(\theta+\chi)^{2}+\left(k^{2} \mu-\nu\right)^{2}}, \\
\tau_{G} & =T\left(\alpha_{G r} \frac{\partial \alpha_{G i}}{\partial \omega}-\alpha_{G i} \frac{\partial \alpha_{G r}}{\partial \omega}\right) .
\end{aligned}
$$

In Figure 2 we plot the transmission coefficient $T_{G}$, for the same parameter values used in Figure 1. The qualitative and quantitative agreements are good. We do not present the phase time of (28) because the evaluation, using Mathematica code, diverges for Bragg-grating lengths of the order of $10 \mu$. To overcome this difficulty one can combine this Mathieu functions approach with the theory of finite periodic systems. The results of this combined approach will be shown after discussing the results obtained in the TFPS.

2.3. The FBG in the TFPS. To simplify the calculation we shall assume that the refractive index is $r(z)=r_{o}(1+q(z))$ with $q(z)$, a sectionally constant periodic function defined as

$$
q(z)= \begin{cases}\frac{V_{o}}{2} & 0<z \bmod (\Lambda) \leq \frac{\Lambda}{2} \\ \frac{-V_{o}}{2} & \frac{\Lambda}{2}<z \bmod (\Lambda) \leq \Lambda .\end{cases}
$$

In this case the unit cell transfer matrix is

$$
M(\Lambda, 0)=\left(\begin{array}{cc}
\alpha & \beta \\
\beta^{*} & \alpha^{*}
\end{array}\right)
$$

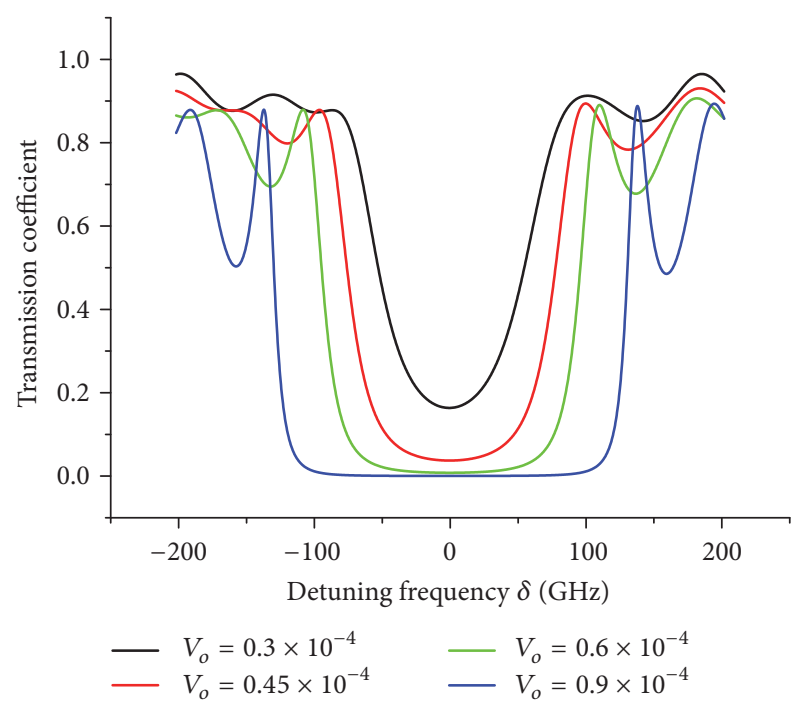

FIgURE 2: The transmission coefficient as a function of the detuning frequency, evaluated with (27) and the Mathieu functions, for different values of the modulation amplitude $V_{o}$ and the same parameter values used in Figure 1. The behavior compares quite well with Figure 1; however the phase time using a code like Mathematica diverges for BG length above $L_{o} \simeq 10 \mu$.

with the real and imaginary parts of $\alpha$ and $\beta$ given by

$$
\begin{aligned}
\alpha_{R}= & \cos \left(k r_{1} a\right) \cos \left(k r_{2} b\right) \\
& -\frac{r_{1}^{2}+r_{2}^{2}}{2 r_{1} r_{2}} \sin \left(k r_{1} a\right) \sin \left(k r_{2} b\right), \\
\alpha_{I}= & \sin \left(k r_{1} a\right) \cos \left(k r_{2} b\right) \\
& +\frac{r_{1}^{2}+r_{2}^{2}}{2 r_{1} r_{2}} \cos \left(k r_{1} a\right) \sin \left(k r_{2} b\right), \\
\beta_{I}= & -\frac{r_{1}^{2}-r_{2}^{2}}{2 r_{1} r_{2}} \sin \left(k r_{2} b\right), \\
\beta_{R}= & 0 .
\end{aligned}
$$

Here $a=b=\Lambda / 2$ are the half-cell lengths with refractive indices $r_{1}=r_{0}-V_{o} / 2$ and $r_{2}=r_{0}+V_{o} / 2$, respectively. In the theory of finite periodic systems, the transfer matrix of the whole $n$-cells Bragg grating is [21]

$$
M(n \Lambda, 0)=\left(\begin{array}{ll}
\alpha_{n} & \beta_{n} \\
\beta_{n}^{*} & \alpha_{n}^{*}
\end{array}\right)
$$

with

$$
\begin{aligned}
& \alpha_{n}=U_{n}\left(\alpha_{R}\right)-\alpha^{*} U_{n-1}\left(\alpha_{R}\right) . \\
& \beta_{n}=\beta U_{n-1}\left(\alpha_{R}\right)
\end{aligned}
$$

where $U_{n}\left(\alpha_{R}\right)$ is the Chebyshev polynomial of the second kind and order $n$, evaluated at $\alpha_{R}$. The transmission 


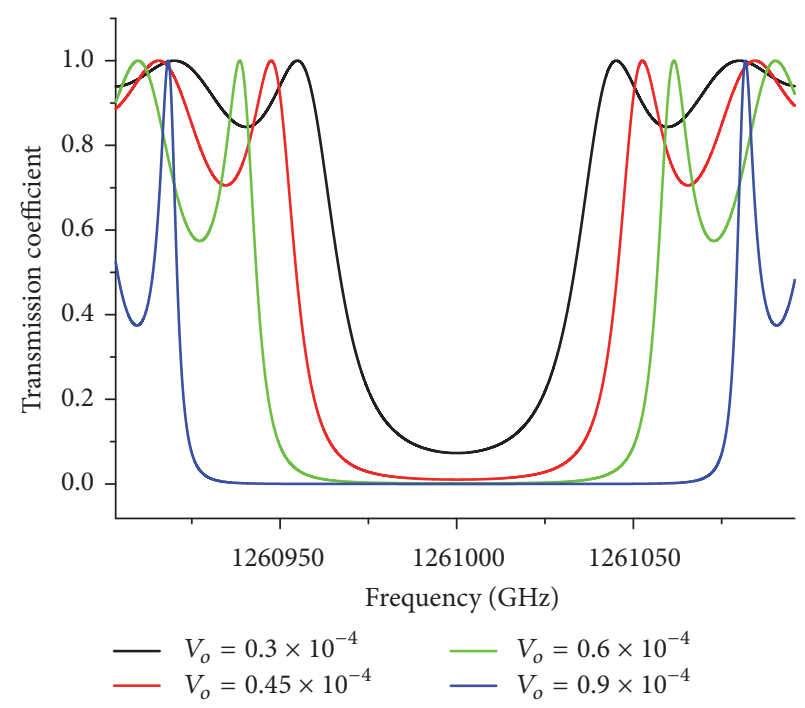

(a)

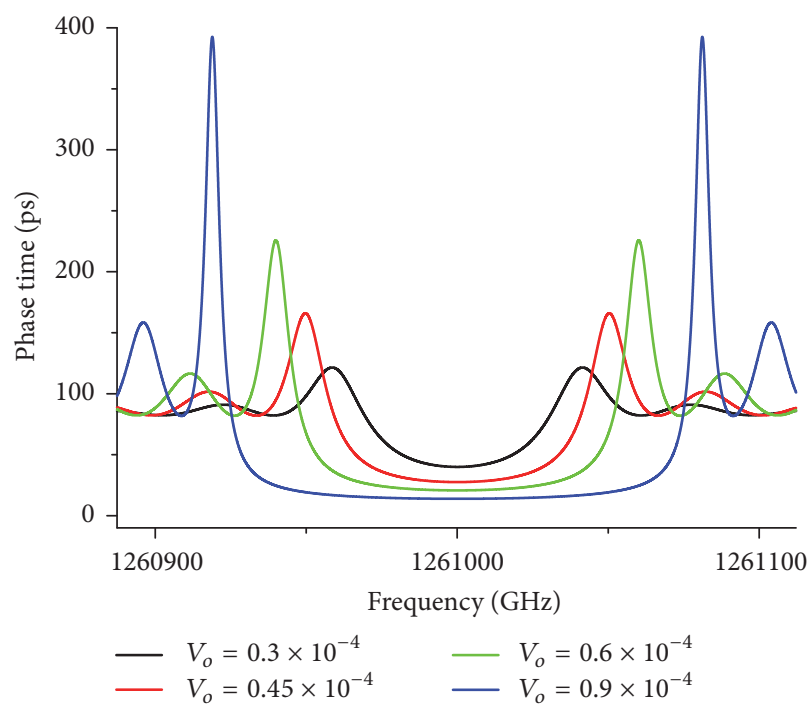

(b)

FIGURE 3: The transmission coefficient (a) and the phase time (b) from the TFPS, as functions of the frequency, for different values of the "modulation amplitude" $V_{o}$ with $w_{B}=1.261 \times 10^{15} \mathrm{~Hz}, r_{0}=1.452$, and $L_{o}=8.5 \mathrm{~mm}$.

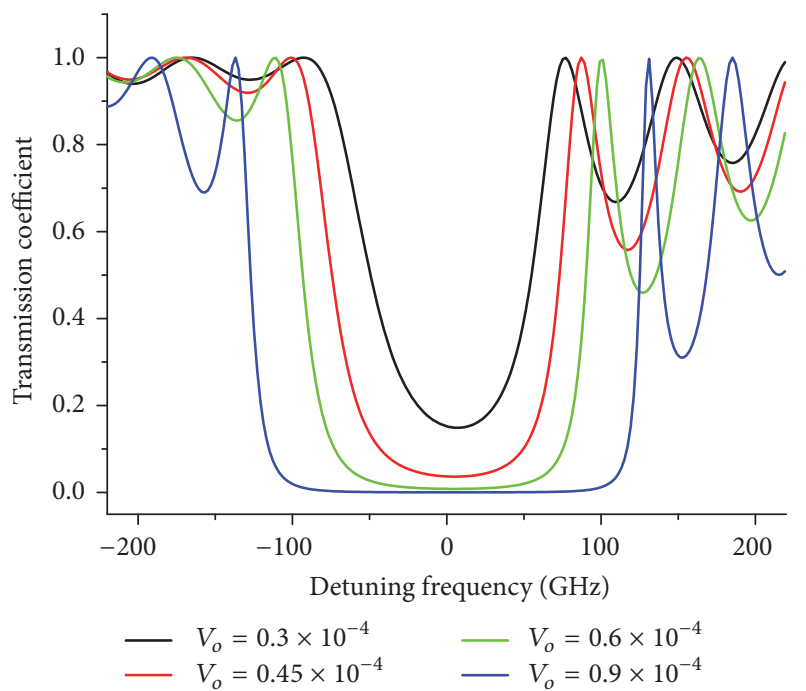

(a)

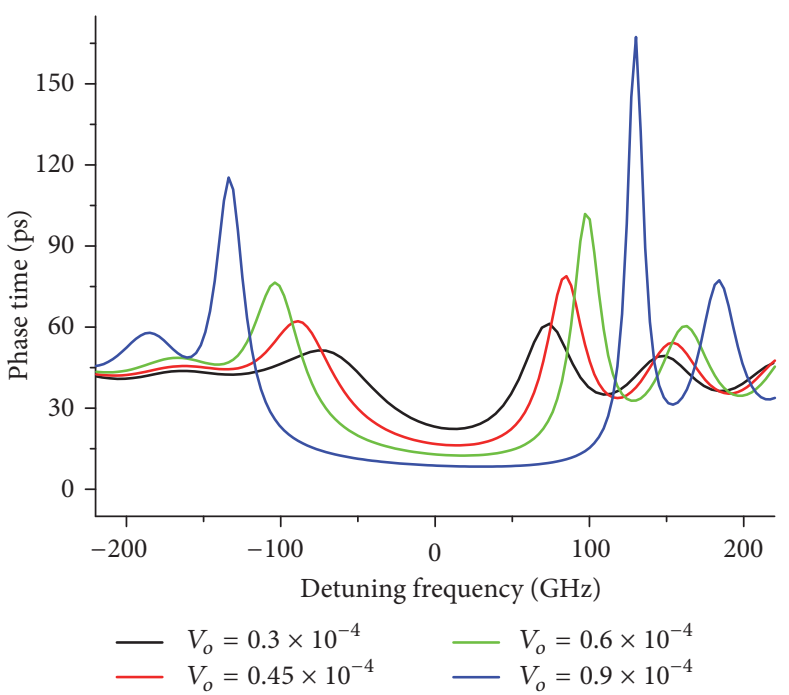

(b)

FIGURE 4: The transmission coefficient (a) and the phase time (b) from a combined Mathieu functions and FPS theory, as functions of the detuning frequency, for different values of the "modulation amplitude" $V_{o}$ with $w_{B}=1.261 \times 10^{15} \mathrm{~Hz}, r_{0}=1.452, n=3445$, and $L_{o}=8.5 \mathrm{~mm}$.

coefficient and the phase time of the $n$-cells Bragg grating can be evaluated from

$$
\begin{gathered}
T_{n}=\frac{1}{\left|\alpha_{n}\right|^{2}}=\frac{1}{\left|U_{n}-\alpha^{*} U_{n-1}\right|^{2}}, \\
\tau_{n}(E)=-a \hbar \frac{d k}{d E}+\frac{\hbar}{\left|\alpha_{n}\right|^{2}}\left\{\frac{1}{2} U_{2 n-1}\left(\alpha_{R}\right) \frac{d \alpha_{I}}{d E}\right. \\
\left.\quad-\frac{\alpha_{I}}{\left(1-\alpha_{R}^{2}\right)}\left[n-\frac{\alpha_{R}}{2} U_{2 n-1}\left(\alpha_{R}\right)\right] \frac{d \alpha_{R}}{d E}\right\} .
\end{gathered}
$$

In Figure 3 we plot these quantities for the same parameters as in Figure 1. There is also a very good qualitative and quantitative agreement with the results in Figure 1.

2.4. The FBG Combining Mathieu Functions and the TFPS. To avoid divergencies when standard codes like Mathematica are used to evaluate Mathieu functions and their derivatives, one can divide the Bragg grating of length $L_{o}$ by some natural number $n \gtrsim 1000$ such that the Mathieu functions evaluated at the new BG length $l_{o}=L_{o} / n$ is congruent with $\Lambda$. The smaller Bragg gratings can now be taken as the unit cell in the TFPS. For $n$ a multiple of 689 with $w_{B}=\pi c / r_{o} \Lambda=$ 
$1.2618710^{15} \mathrm{~Hz}$ and $r_{o}=1.452$ we obtain the transmission coefficients and phase times shown in Figure 4, for the same values of $V_{o}$ as in Figures 1,2 and 3. The qualitative and quantitative agreement is really good.

The theory of finite periodic systems and the Mathieu functions approach were also applied to study the double Bragg grating, particularly to understand the effect of the Bragg gratings separation in the tunneling time behavior and to compare with other approaches' predictions [27].

2.5. Conclusions. We have shown that the transmission of electromagnetic waves, through fiber Bragg gratings, can be faithfully studied using the theory of finite periodic system either alone or combined with the Mathieu functions.

\section{Competing Interests}

The author declares that there is no conflict of interests regarding the publication of this paper.

\section{References}

[1] A. Othonos and K. Kalli, Fiber Bragg Gratings: Fundamentals and Applications in Telecommunications and Sensing, Artech House, 1999.

[2] P. Ferraro and G. De Natale, "On the possible use of optical fiber Bragg gratings as strain sensors for geodynamical monitoring," Optics and Lasers in Engineering, vol. 37, no. 2-3, pp. 115-130, 2002.

[3] T. Guo, X. Qiao, Z. Jia, Q. Zhao, and X. Dong, "Simultaneous measurement of temperature and pressure by a single fiber Bragg grating with a broadened reflection spectrum," Applied Optics, vol. 45, no. 13, pp. 2935-2939, 2006.

[4] V. V. Spirin, M. G. Shlyagin, S. V. Miridonov, and I. Marquez, "Temperature-insensitive strain measurement using differential double Bragg grating technique," Optics and Laser Technology, vol. 33, no. 1, pp. 43-46, 2001.

[5] N. Tanaka, Y. Okabe, and N. Takeda, "Temperature-compensated strain measurement using fiber Bragg grating sensors embedded in composite laminates," Smart Materials and Structures, vol. 12, no. 6, pp. 940-946, 2003.

[6] A. Sun, X. G. Qiao, Z. A. Jia, M. Li, and D. Z. Zhao, "Study of simultaneous measurement of temperature and pressure using double fiber Bragg gratings with polymer package," Optical Engineering, vol. 44, no. 3, Article ID 034402, 2005.

[7] O. Frazão, L. A. Ferreira, F. M. Araújo, and J. L. Santos, "Applications of fiber optic grating technology to multi-parameter measurement," Fiber and Integrated Optics, vol. 24, no. 3-4, pp. 227-244, 2005.

[8] M. Attygalle, C. Lim, G. J. Pendock, A. Nirmalathas, and G. Edvell, "Transmission improvement in fiber wireless links using fiber Bragg gratings," IEEE Photonics Technology Letters, vol. 17, no. 1, pp. 190-192, 2005.

[9] H. Kogelnik, "Coupled wave theory for thick hologram gratings," Bell System Technical Journal, vol. 48, no. 9, pp. 2909-2947, 1969.

[10] A. Yariv, "Coupled-mode theory for guided-wave optics," IEEE Journal of Quantum Electronics, vol. 9, no. 9, pp. 919-933, 1973.
[11] A. W. Snyder, "Coupled-mode theory for optical fibers," Journal of the Optical Society of America, vol. 62, no. 11, pp. 1267-1277, 1972.

[12] D. Marcuse, "Coupled mode theory of round optical fibers," The Bell System Technical Journal, vol. 52, no. 6, pp. 817-842, 1973.

[13] W. Streifer, D. Scifres, and R. Burnham, "TM-mode coupling coefficients in guided-wave distributed feedback lasers," IEEE Journal of Quantum Electronics, vol. 12, no. 2, pp. 74-78, 1976.

[14] H. Kogelnik and C. V. Shank, "Coupled-wave theory of distributed feedback lasers," Journal of Applied Physics, vol. 43, no. 5, pp. 2327-2335, 1972.

[15] H. Kogelnik, E. Garmire, and J. M. Hammer, Integrated Optics, Spriger, Berlin, Germany, 1st edition, 1975.

[16] T. Erdogan, "Fiber grating spectra," Journal of Lightwave Technology, vol. 15, no. 8, pp. 1277-1294, 1997.

[17] F. Abeles, "Sur la propagation des ondes electromagnetiques dans les milieux stratifies," Annales de Physique (Paris), vol. 5, pp. 504-520, 1950.

[18] M. Born and E. Wolf, Principles of Optics, Dover, Pergamon, Oxford, UK, 1980

[19] M. Pacheco and F. Claro, "Simple results for one-dimensional periodic potentials," Physica Status Solidi (B), vol. 114, no. 2, pp. 399-403, 1982.

[20] D. J. Griffiths and N. F. Taussig, "Scattering from a locally periodic potential," American Journal of Physics, vol. 60, no. 10, pp. 883-888, 1992.

[21] P. Pereyra, "Resonant tunneling and band mixing in multichannel superlattices," Physical Review Letters, vol. 80, no. 12, pp. 2677-2680, 1998.

[22] P. Pereyra and E. Castillo, "Theory of finite periodic systems: general expressions and various simple and illustrative examples," Physical Review B, vol. 65, no. 20, Article ID 205120, 2002.

[23] P. Pereyra and H. P. Simanjuntak, "Time evolution of electromagnetic wave packets through superlattices: evidence for superluminal velocities," Physical Review E, vol. 75, no. 5, Article ID 056604, 2007.

[24] S. Longhi, P. Laporta, M. Belmonte, and E. Recami Phys, "Critical number of atoms for attractive Bose-Einstein condensates with cylindrically symmetrical traps," Physical Review E, vol. 64, no. 5, Article ID 055602, 2001.

[25] P. M. Morse and H. Feshbach, Methods of Theoretical Physics, Part I, McGraw Hill, New York, NY, USA, 1953.

[26] A. Anzaldo-Meneses and P. Pereyra, "Sylvester theorem and the multichannel transfer matrix method for arbitrary transverse potential profile inside a wave guide," Annals of Physics, vol. 322, no. 9, pp. 2114-2128, 2007.

[27] H. P. Simanjuntak and P. Pereyra, "On the generalized Hartman effect presumption in semiconductors and photonic structures," Nanoscale Research Letters, vol. 8, article no. 145, 2013. 

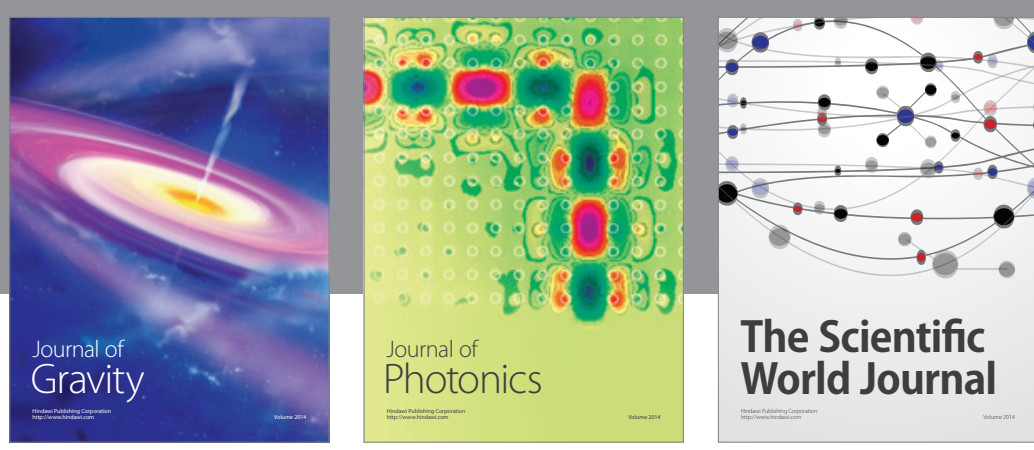

The Scientific World Journal
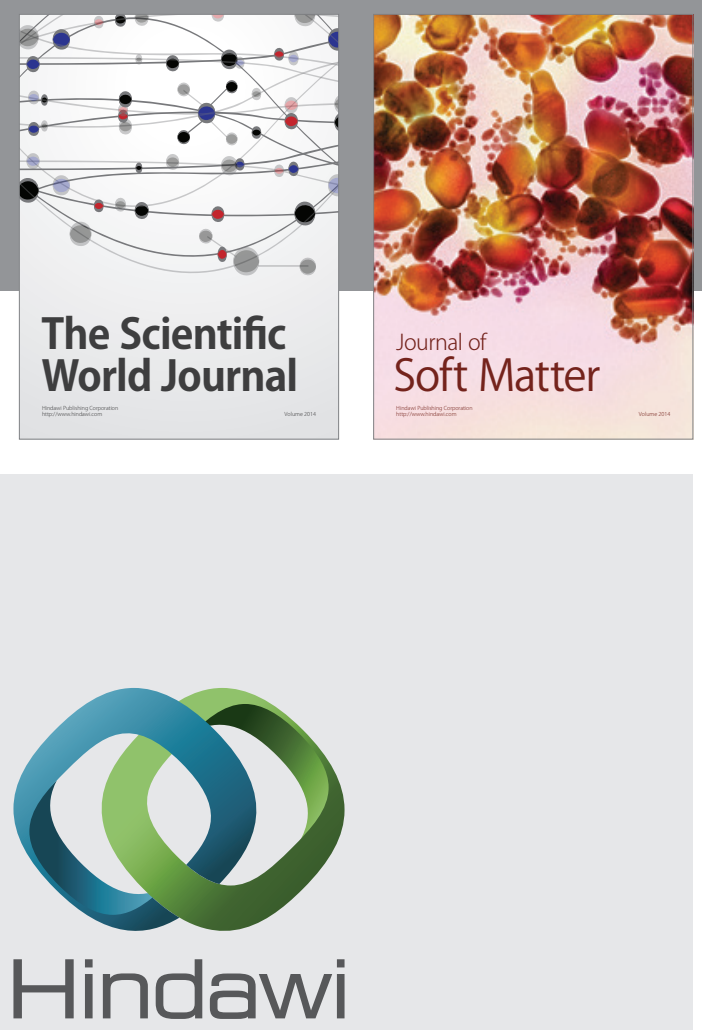

Submit your manuscripts at

https://www.hindawi.com
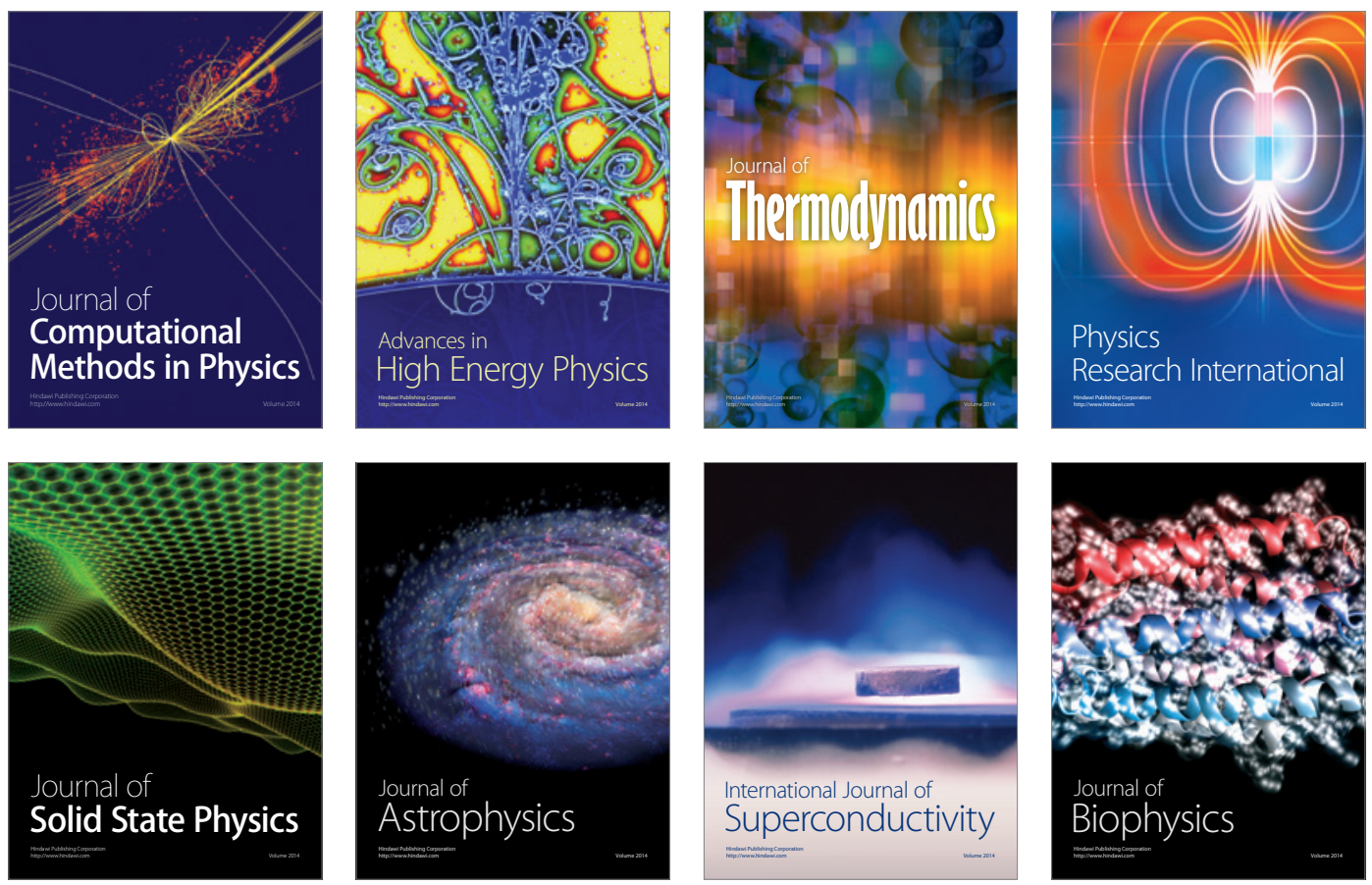
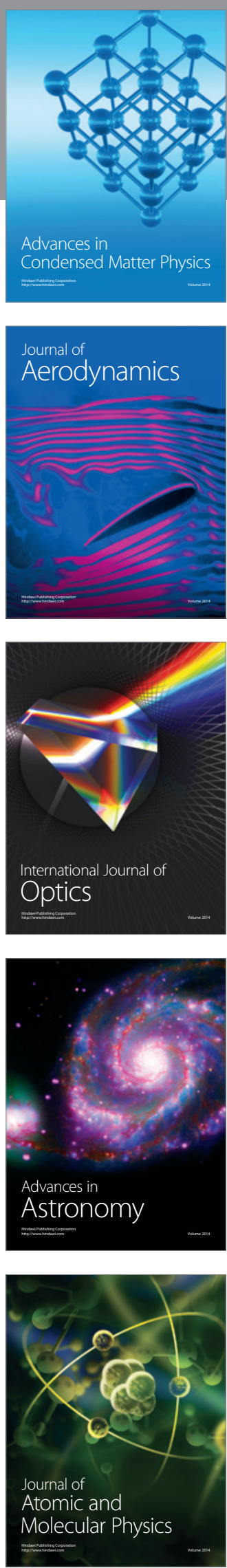